\section{Tea as a Source of Acinetobacter baumannii Ventilator-Associated Pneumonia?}

\author{
To the Editor: \\ Acinetobacter baumannii increas- \\ ingly has been involved as an agent of \\ hospital outbreaks worldwide. The \\ most common site of nosocomial \\ infections is the respiratory tract, \\ especially in ventilated patients, fol- \\ lowed by bloodstream infections. ${ }^{1}$ The \\ incidence of Acinetobacter species as \\ a cause of nosocomial pneumonia has \\ increased despite advances in the \\ management of ventilated patients \\ and the use of better disinfection pro- \\ cedures for ventilatory equipment. \\ Spread of Acinetobacter via hands of \\ staff members was found to be impor- \\ tant in many studies. ${ }^{2}$ Outbreaks due \\ to contaminated medical equipment \\ and materials, including mattresses \\ and pillows, also have been reported, ${ }^{3}$ \\ but tea or other fluids used for mouth \\ hygiene have not previously been \\ reported as a source of infections \\ caused by Acinetobacter species. \\ Between October 1997 and \\ October 1998, A baumannii (identified \\ by API $20 \mathrm{NE}$, bioMérieux, Nürtingen,
}

Germany) was isolated in our neurological intensive-care unit from tracheal secretions (39 patients), a cerebrospinal fluid specimen, and a skin swab. All isolates but one were sensitive to cotrimoxazole. Ventilatorassociated pneumonia caused by $A$ baumannii, alone or in combination with other pathogens, was diagnosed in 18 patients. There was one case of relapsing Acinetobacter ventriculoperitoneal shunt infection.

Genotyping by randomly amplified polymorphic DNA-polymerase chain reaction revealed 10 genetically different strains, 6 of which were involved in small clusters of colonized or infected patients. ${ }^{4}$

Sampling of 288 environmental sites (including hands of personnel) revealed three strains isolated from tapwater and from anti-splash nozzles that were identical to those found in three patients. Contaminated respiratory equipment or contamination of the patients' inanimate environment could not be identified. One of the outbreak strains also was isolated from tea used for mouth care. Native tea was found to be heavily contaminated with a wide range of gram-negative bacteria, but not with $A$ baumannii.

Contamination of the brewed tea may have occurred due to mixing the hot tea with tap water to cool it or to brewing with tap water insufficiently heated in an automatic water heater.

Our findings raise the possibility of the acquisition of $A$ baumannii from tea used for mouth hygiene.

\section{REFERENCES}

1. Forster DH, Daschner FD. Acinetobacter species as nosocomial pathogens. Eur J Clin Microbiol Infect Dis 1998;17:73-77.

2. Go SE, Urban C, Burns J, Kreiswirth B, Eisner W, Mariano N, et al. Clinical and molecular epidemiology of Acinetobacter infections sensitive only to polymyxin B and sulbactam. Lancet 1994;344:1329-1332.

3. Bergogne-Bérézin E, Towner KJ. Acinetobacter spp as nosocomial pathogens: microbiological, clinical and epidemiological features. Clin Microbiol Rev 1996;9:148-165.

4. Webster CA, Towner KJ, Humphreys $\mathrm{H}$, Ehrenstein B, Hartung D, Grundmann $\mathrm{H}$ Comparison of rapid automated laser fluorescence analysis of DNA fingerprints with four other computer-assisted approaches for studying relationships between Acinetobacter baumannii isolates. $J$ Med Microbiol 1996;44:185-194.

Thomas Hauer, MD

Daniel Jonas, MD

Markus Dettenkofer, MD

Franz D. Daschner, MD

Institute for Environmental Medicine and Hospital Epidemiology University Hospital of Freiburg Freiburg, Germany

\title{
Vancomycin Use in 41 US Hospitals
}

\section{Gina Pugliese, RN, MS} Martin S. Favero, PhD

The emergence of vancomycinresistant Staphylococcus aureus in the United States and Japan has reintensified efforts to use antimicrobials more judiciously, but coming up with effective ways to reduce the use of vancomycin in hospitals has proven to be a tough challenge. To evaluate antimicrobial use across the country, Fridkin and colleagues surveyed 108 adult ICUs in 41 US hospitals to find out how much van- comycin they used during 1996 to 1997 , as well as what proportion of their total $S$ aureus isolates were resistant to methicillin.

The median rate of vancomycin use was found to be lowest in coronary care ICUs and highest in surgical ICUs. Approximately only one quarter of the hospitals required prior approval for the use of vancomycin. Also, rates of methicillin-resistance, central-line-associated bloodstream infections, and type of ICU all were independent predictors of vancomycin use. The researchers note that not a single infection control practice was associated with reduced use of vancomycin.

The authors conclude that the use of vancomycin is determined mostly by the endemic extent of methicillin-resistant organisms and central-line-associated infections. They emphasize that hospitals trying to reduce the use of vancomycin need to focus on further improving their infection control practices.

FROM: Fridkin SK, Edwards JR, Pichette SC. Determinants of vancomycin use in adult intensive care units in 41 United States hospitals. Clin Infect Dis 1999;28:1119-1125. 